\title{
On the Interaction of Resonance And Bragg Scattering Effects for the Locally Resonant Phononic Crystal with Alternating Elastic and Fluid Matrices
}

\author{
Bo YUAN(1), Yong $\mathrm{CHEN}^{(2)}$, Min JIANG ${ }^{(1)}$, Shuai TANG(1), Miao HE ${ }^{(3)}$, Minglin TU(1) \\ (1) Department of Machinery and Electrical Engineering \\ Army Logistics University of PLA \\ 401311, Chongqing, China; e-mail: ramboyuanbo@aliyun.com \\ ${ }^{(2)}$ College of Aerospace Science and Engineering \\ National University of Defense Technology \\ 410073, Changsha, China \\ (3) Department of Military Engineering Management \\ Army Logistics University of PLA \\ 401311, Chongqing, China
}

(received July 12, 2016; accepted June 21, 2017)

\begin{abstract}
Three-dimensional (3D) locally resonant phononic crystals (LRPCs) are studied with the aim of optimising the sub-wavelength band gaps of such composites. By analysing their effective acoustic properties, it has been found that the effective acoustic speed of the composite will drop to zero when local resonance arise, and will increase monotonically when Bragg scattering effects occur. Moreover, if the matrix is a low-shear-speed medium, local resonators can significantly reduce the effective acoustic speed of the composite and, therefore, lower the frequency where Bragg scattering effects occur. Hence, a specific LRPC with alternating elastic and fluid matrices is proposed, whose resonance and Bragg gaps are already close in frequency. The fluid matrix behaves as a wave filter, which prevents the shear waves from propagating in the composite. By using the layer-multiple-scattering theory, the coupling behaviour of local resonance and Bragg scattering band gaps has been investigated. Both gaps are enhanced when they move closer to each other. Finally, a gap-coupled case is obtained that displays a broad sub-wavelength band gap. Such proposal excels at the application of underwater acoustic materials since the arrangement of structure can be handily adjusted for tuning the frequency of coupled gap.
\end{abstract}

Keywords: locally resonant phononic crystals; effective speed of sound; coupling band gaps; underwater acoustic materials.

\section{Introduction}

The phononic crystal (PC) is a kind of artificial composite with structural periodicities, which can control the propagation of acoustic wave by Bragg band gaps. Such gaps are induced by the constructive interference of scattered waves from different scatterers inside the composite (Sigalas, Economou, 1992; Kushwaha et al., 1993). In the past decade, a number of researchers have studied the locally resonant phononic crystals (LRPCs) since it can exhibit an obvious sub-wavelength band gap with structural periodicity two orders of magnitude smaller than the relevant sonic wavelength (GofFAux et al.,
2002; Liu et al., 2002; Goffaux, SÁnchez-Dehesa, 2003). Such composites are improved by substituting the rigid scatterers in PCs with local resonators (LRs) which can vibrate fiercely in proper excitation (LiU et al., 2000; 2005). Particularly, three-dimensional (3D) LRPCs have great prospects for the application of low-frequency underwater acoustic materials (ZHAO et al., 2007; Wen et al., 2011).

Since the fundamental resonances of LRs are usually confined to the low frequency region, most studies have focused on the existence of resonance gaps, as well as the transmission or absorption performance of local resonance (HIRSEKORN, 2004; ZHAO et al., 2005; WANG et al., 2006). The Bragg scattering behaviour in 
LRPCs is usually neglected as such phenomena usually occur in a higher frequency range. Hence, the periodic arrangements of LRs have not been fully utilized (LIU et al., 2002). On the other hand, LRPCs have been suffering from the narrowness of resonance gaps during their application since resonators will only response strongly when the excitation is close to their intrinsic frequencies (LARABI et al., 2007; ElFORD et al., 2011).

To broaden the sub-wavelength band gaps of LRPCs, we tried to couple the resonance and Bragg scattering band gaps together. In this respect, XIAO et al. (2011) studied the coupling of resonance and Bragg band gaps in a one-dimensional (1D) locally resonant elastic system (LRES). Such LRES consisted of a taut uniform string with periodically attached spring-mass resonators. The analysis demonstrated that both types of gaps can be enhanced when they are close to each other in frequency or coupled together. However, their system was based on ideal 1D discrete periodic structure. The resonance or the Bragg scattering frequencies can be tuned individually by varying the stiffness of spring or the periodicity of resonators. In regard to the 3D LRPC, it will be more complicated to tune the frequency of band gap.

In this paper, the acoustic behaviour of resonance and Bragg scattering effects and the influence of LRs on the effective speeds of LRPCs are analysed. A 3D LRPC with alternating fluid and elastic matrices are proposed, which has the potential for the coupling of different band gaps. The coupling behaviours of resonance and Bragg scattering gaps are discussed, with the aim of generating wide low-frequency band gaps.

\section{Configuration and modelling}

A 3D LRPC typically consists of infinite local resonators (LRs) periodically embedded in a fluid or elastic matrix. The LRs are usually dense metallic spheres coated with soft rubber. There are several different lattices to array the resonators in the matrix (KUANG et al., 2004), while only cuboid cells are considered in this paper. Hence, no offsets exist between the different layers of LRs. Figure 1 shows a section view of such a 3D LRPC with the cutting plane crossing through the centres of LRs. In the schematic diagram, black circles indicate the resonators' cores; the white annuluses around cores represent the soft coats; the grey background represents the elastic matrix; while the dashed lines in the matrix demonstrate boundaries of different layers of LRs. The resonators in each layer are arranged periodically on a square lattice, whose unit vectors lie parallel to the $x$ - and $y$-directions. The third unit vector is normal to the surface of LRs layer. The periodicity of the structure in the $z$-direction is called the layer thickness $(L)$, while periodicities in $x$ - and $y$-directions are named as the lattice constant $(A)$.

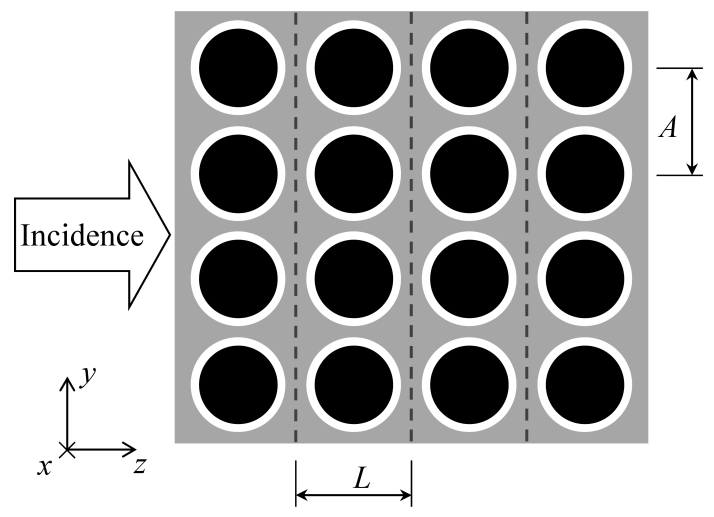

Fig. 1. A section view of the 3D LRPC with the cutting plane crossing through the centres of LRs.

Consider a 3D LRPC with a natural rubber matrix, whose mass density is $\rho_{\mathrm{M}}=1300 \mathrm{~kg} \cdot \mathrm{m}^{-3}$, and the speeds of longitudinal and shear waves in the matrix are $c_{\mathrm{ML}}=1510 \mathrm{~m} \cdot \mathrm{s}^{-1}$ and $c_{\mathrm{MS}}=783 \mathrm{~m} \cdot \mathrm{s}^{-1}$. LRs are periodically embedded in the matrix with a layer thickness $(L)$ of $32 \mathrm{~mm}$ and a lattice constant $(A)$ of $16 \mathrm{~mm}$. The resonators are set to be lead spheres, $8 \mathrm{~mm}$ in diameter, coated with silicone rubbers whose thicknesses are $1 \mathrm{~mm}$. The acoustic properties of the lead are $\rho_{\mathrm{S}}=11600 \mathrm{~kg} \cdot \mathrm{m}^{-3}, c_{\mathrm{SL}}=2493 \mathrm{~m} \cdot \mathrm{s}^{-1}$ and $c_{\mathrm{SS}}=1133 \mathrm{~m} \cdot \mathrm{s}^{-1}$, while those of the silicone rubber are $\rho_{\mathrm{C}}=1039 \mathrm{~kg} \cdot \mathrm{m}^{-3}, c_{\mathrm{CL}}=216 \mathrm{~m} \cdot \mathrm{s}^{-1}$ and $c_{\mathrm{CS}}=63 \mathrm{~m} \cdot \mathrm{s}^{-1}$. Such configuration is named as Case I. All the materials used in this paper are lossless as we mainly discuss the effective acoustic speed and the band structure of LRPCs.

The preliminary goal of this paper is to investigate the dynamic effective acoustic speeds of an LRPC. Most methods used for calculating the effective properties of acoustic metamaterials are based on an assumption - the long wavelength limitation - that the wavelength of sound at the frequencies of interest should be much longer than the periodicity of structure (ZHOU, Hu, 2009). This assumption is no longer adapted to the Bragg frequency region, since the wavelength is comparable with the periodicity of the structure when Bragg scattering effect happens. Hence, in the present paper, the effective speed of sound $\left(c_{\mathrm{E}}\right)$ is retrieved from the complex reflection and transmission coefficients of a finite slab of LRPC following the approach given by FOKIN et al. (2007), which is designated as the retrieval method (RM).

In this approach, we first use the layer-multiplescattering theory (LMST) (SAINIDOU et al., 2005) to calculate the complex reflection $(R)$ and transmission $(T)$ coefficients of a finite LRPC for normal incidence. Then, these coefficients are used to retrieve the composite's equivalent relative impedance $(\xi=$ $\left.\rho_{\mathrm{E}} c_{\mathrm{E}} / \rho_{\mathrm{M}} c_{\mathrm{M}}\right)$ and refractive index $\left(n=c_{\mathrm{M}} / c_{\mathrm{E}}\right)$, so as to obtain the material's effective mass density $\left(\rho_{\mathrm{E}}\right)$ 
and effective speed of sound $\left(c_{\mathrm{E}}\right)$. Note that the $\rho_{\mathrm{E}}$ and $c_{\mathrm{E}}$ are complex values, whose imaginary parts imply the extents of attenuation when the propagating waves are evanescent in the composite. $\rho_{\mathrm{M}}$ and $c_{\mathrm{M}}$ are the mass density and the speed of sound of the matrix. According to FOKIN et al. (2007), the equivalent relative impedance $(\xi)$ and the refractive index $(n)$ of the composite are obtained from:

$$
\xi=\frac{r}{1-2 R+R^{2}-T^{2}}, \quad n=\frac{-i \log x+2 \pi m}{k d},
$$

where

$$
\begin{aligned}
& x=\frac{1-R^{2}+T^{2}+r}{2 T}, \\
& r= \pm \sqrt{\left(R^{2}-T^{2}-1\right)^{2}-4 T^{2}} .
\end{aligned}
$$

Here, $k=\omega / c_{\mathrm{M}}$ is the wave number in the matrix, $d$ is the thickness of the simulated LRPC, and $m$ is the branch number of the $\cos ^{-1}$ function, which has been transformed to the ' $-i \log x$ ' term. For a passive sonic material, the real part of $\xi$ should remain positive, which determines the sign of $r$. The term ' $-i \log x+2 \pi m$ ' in Eq. (1) represents the complex phase delay of waves travelling through the slab of LRPC, which can be verified by the definition of phase delay to be $\phi=2 \pi f d / c_{\mathrm{E}}=\left(c_{\mathrm{M}} / c_{\mathrm{E}}\right) \cdot\left(2 \pi f / c_{\mathrm{M}}\right) \cdot d=$ $n k d$. The selection of the branch number $m$ is crucial for evaluating the correct $c_{\mathrm{E}}$. It should take an appropriate integral value determined by the continuity of refractive index $n$. The $R$ and $T$ are the complex reflection and transmission coefficients of one layer of such LRPC which are calculated by LMST. Another notice which should be pointed out is that the $c_{\mathrm{E}}$ will in general be a function of direction; that evaluated by $\mathrm{RM}$ in this paper will be for waves propagating in the $z$-direction.

In the following, the $c_{\mathrm{E}}$ obtained from RM will be compared with the result evaluated by the effectivemedium theory (EMT) (ZHou, Hu, 2009). This is a homogenization theory based on the long wavelength hypothesis, which gives a good prediction in the lowfrequency region. As the EMT only gives out the complex effective mass density $\left(\rho_{\mathrm{E}}\right)$, bulk modulus $\left(\kappa_{\mathrm{E}}\right)$ and shear modulus $\left(\mu_{\mathrm{E}}\right)$ of an LRPC, we extend them to complex effective longitudinal and shear speeds by using $c_{\mathrm{L}}=\sqrt{\left(\kappa_{\mathrm{E}}+4 \mu_{\mathrm{E}} / 3\right) / \rho_{\mathrm{E}}}$ and $c_{S}=\sqrt{\mu_{\mathrm{E}} / \rho_{\mathrm{E}}}$.

\section{Investigation of the effective speed of sound in LRPCs}

Based on RM and EMT, the complex effective speeds of longitudinal and shear waves in the LRPC are evaluated in this section. Their real parts $\left(c_{\mathrm{EL}}\right.$ for longitudinal waves and $c_{\mathrm{ES}}$ for shear waves) are shown in Fig. 2. For convenience, the effective speeds are plotted as a function of the non-dimensional frequency
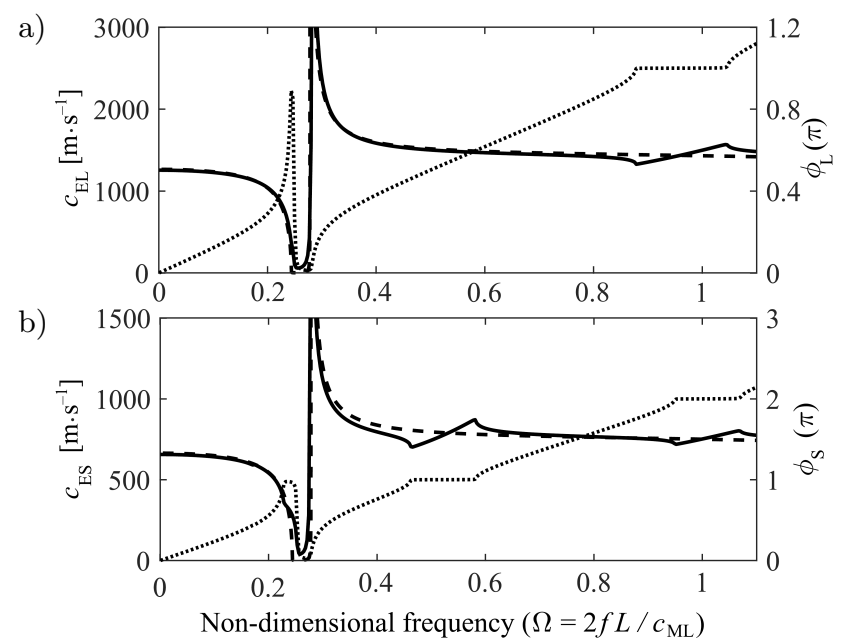

Fig. 2. a) The effective speed of longitudinal waves $\left(c_{\mathrm{EL}}\right)$, b) the effective speed of shear waves $\left(c_{\mathrm{ES}}\right)$, and the phase delays per layer for both wave modes $\left(\phi_{\mathrm{L}}\right.$ and $\left.\phi_{\mathrm{S}}\right)$. The solid and dashed curves in both subfigures represent the $c_{\mathrm{E}}$ evaluated by RM and EMT respectively, while the dotted curves indicate the phase delays per layer of corresponding waves obtained from RM.

$\Omega=2 f L / c_{\mathrm{ML}}$, where $c_{\mathrm{ML}}$ is the speed of longitudinal waves in the matrix $\left(1510 \mathrm{~m} \cdot \mathrm{s}^{-1}\right)$, and $L$ is the layer thickness $(32 \mathrm{~mm})$. When $\Omega=1$, the wavelength of longitudinal waves in the matrix is twice of the layer thickness, which occurs at $23.6 \mathrm{kHz}$ for Case I. It needs to be noted that the non-dimensional frequency for $c_{\mathrm{ES}}$ still uses the longitudinal speed of matrix $\left(c_{\mathrm{ML}}\right)$ as reference, so that the acoustic behaviour of both wave modes can be compared on the same frequency scale.

As shown in Fig. 2a, the $c_{\mathrm{EL}}$ evaluated by RM (the solid curve) exhibits acoustic characteristics of both local resonance and Bragg scattering effects over the displayed frequency range. First of all, in the frequency region $\Omega \in[0,0.40], c_{\mathrm{EL}}$ displays highly asymmetrical variations, and the $\phi_{\mathrm{L}}$ encounters a $\pi$ jump when $\Omega=0.24$. These characteristics demonstrate that strong resonances of LRs are stimulated by the longitudinal waves in the matrix around such frequencies. The $\pi$ jump of the phase delay is caused by the transition of resonators from out-of-phase to in-phase responses. The asymmetric profile is produced by the resonant scattering phenomenon, which is known as the Fano resonance (FANO, 1961). Particularly, a zero-phasedelay region can be found around $\Omega=0.26$, where the complex effective speed of longitudinal waves is purely imaginary. This means a forbidden band occurs in this frequency region due to the local resonance (Goffaux et al., 2002). Secondly, in the frequency region of $[0.87,1.05], \phi_{\mathrm{L}}$ retains a value of $\pi$, which implies the reflected waves from different layers interfere constructively, as the phase difference between any two of them is a multiple of $2 \pi$. In other words, the longitudinal waves are subject to a Bragg scattering effect in such region. Accordingly, the $c_{\mathrm{EL}}$ shows a monotonic 
and linear increasing profile. This is because the necessary condition for the Bragg scattering effect is that the phase delay per layer $\left(\phi_{\mathrm{L}}=2 \pi f L / c_{\mathrm{EL}}\right)$ should be a multiple of $\pi$, which requires the $c_{\mathrm{EL}}$ to increase linearly with frequency.

Similarly, the $c_{\mathrm{ES}}$ evaluated by the RM also demonstrates the acoustic characteristics of resonance and Bragg scattering effects, which is shown as the solid curve in Fig. 2b. The local resonance for shear waves occurs in the same frequency region as that for longitudinal waves, which can be verified by the $\phi_{\mathrm{S}}$ possessing a $\pi$ jump when $\Omega=0.24$. However, the Bragg scattering effects for shear waves occur in different frequency regions $[0.48,0.59]$ and $[0.95,1.06]$. Specially, the latter region is induced by the second order interference between resonators, as the $\phi_{\mathrm{S}}$ remains $2 \pi$ in this area.

In addition, the dashed curves in Fig. 2 represent the effective speed of sounds predicted by EMT. This approach has been proved to be reliable in the long wavelength limit (ZHOU, Hu, 2009). By comparing the results in Fig. 2, it can be confirmed that the predictions of RM and EMT agree accurately in resonance regions $\left(\Omega \in[0,0.80]\right.$ for $c_{\mathrm{EL}}$, while $\Omega \in[0,0.35]$ for $\left.c_{\mathrm{ES}}\right)$. However, discrepancies arise between the two results when the Bragg scattering effects occur in the LRPC. This is because EMT ignores the interaction between different scatterers during its formation.

In order to validate the prediction of the RM, the dispersion curves derived from $c_{\mathrm{E}}$ calculated by EMT and RM, as well as the band structure calculated from LMST, are compared in Fig. 3. All the subfigures have the same scale of non-dimensional frequency for their $x$-axes, while their $y$-axes denote the real part of the complex wave-vector's $z$-component which has been reduced to the first Brillouin zone. Since the wave-vectors in Fig. 3ab are derived from the composite's complex effective speed of sound for $z$-direction $\left(K_{z}=2 \pi f / c_{\mathrm{E}}\right)$, the reduced real parts of wave-vectors are expressed as $\operatorname{Re}\left[2 f L / c_{\mathrm{E}}\right]$, while that in Fig. 3c is shown as $\operatorname{Re}\left[K_{z} L / \pi\right]$. Due to the symmetry of the composite in $z$-direction, the negative parts of the band structures are not shown in the figure as they are symmetric with their positive parts.

Figure 3 shows that the EMT succeeds in predicting the resonance features of the LRPC, but fails to predict the Bragg scattering behaviour of such a composite. In contrast, the dispersion curves predicted by RM are consistent with the counterparts calculated by LMST in both resonance and Bragg scattering frequency regions, which demonstrates the correctness of RM's prediction. As the LMST can further indicate band gaps of the composite, it can be verified that the Bragg scattering band gap (located in the region of $[0.96,1.04])$ can only be achieved when strong interferences emerge from both wave modes in the same frequency region.

In the above discussion, although the $c_{\mathrm{E}}$ of the composite varies with frequency owing to the resonance and Bragg scattering effects, the average values of $c_{\mathrm{E}}$ are still approximate to the speeds of sound in the matrix $\left(c_{\mathrm{ML}}=1510 \mathrm{~m} \cdot \mathrm{s}^{-1}, c_{\mathrm{MS}}=783 \mathrm{~m} \cdot \mathrm{s}^{-1}\right)$. Consequently, the Bragg scattering effects appear at much higher frequencies than the resonance. If we want to couple the resonance and Bragg gaps together in sub-wavelength region, it is necessary to reduce the $c_{E}$ of the composite significantly. Otherwise, the composite's thickness needs to be tremendously large, which is

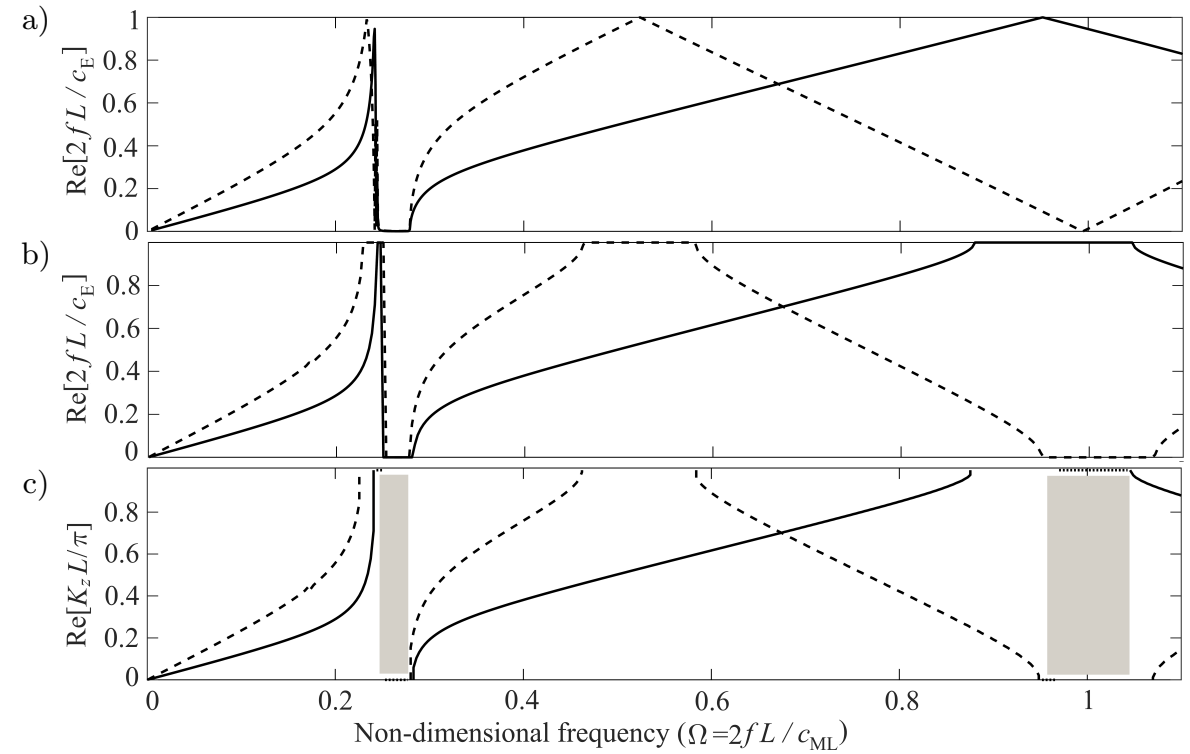

Fig. 3. The dispersion curves of both wave modes derived by EMT (a), RM (b) and LMST (c). The solid (dashed) lines represent the longitudinal (shear) dispersions obtained from the different methods respectively; while the shaded areas in (c) indicate the frequency regions of band gaps produced by local resonance or Bragg scattering effects. 


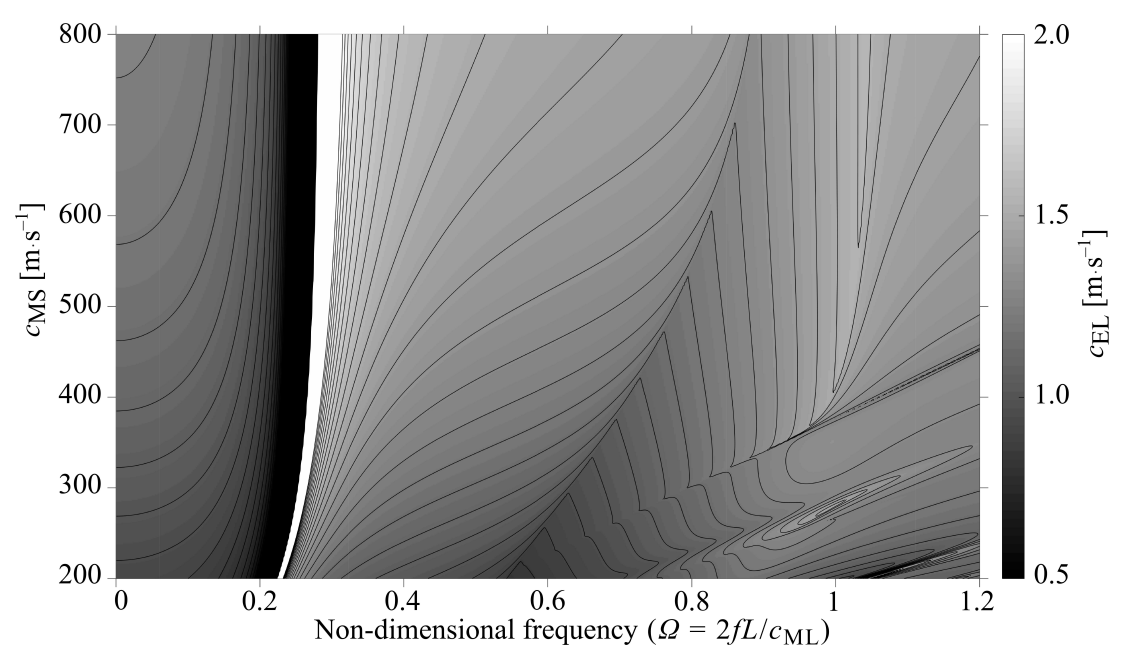

Fig. 4. The contour graph of the effective longitudinal speed $\left(c_{\mathrm{EL}}\right)$ as a function of the shear speed of the matrix $\left(c_{\mathrm{MS}}\right)$ and the non-dimensional frequency $(\Omega)$. The $x$-axis represents the non-dimensional frequency; the $y$-axis indicates the shear speed of the matrix; the grey scale indicates the $c_{\mathrm{EL}}$ of the composite; the solid lines in the graph represent the contour lines of $c_{\mathrm{EL}}$ with an interval between two adjacent levels of $50 \mathrm{~m} \cdot \mathrm{s}^{-1}$.

unpractical for the application of underwater acoustic materials.

Through our research, it is found that the soft coat of LRs can significantly reduce the $c_{\mathrm{E}}$ of the composite for certain LRPCs. Furthermore, it is the shear speed of the matrix $\left(c_{\mathrm{MS}}\right)$ that determines the extent of the LRs' influence. Figure 4 shows a contour graph for the variation of the effective longitudinal speed of the composite $\left(c_{\mathrm{EL}}\right)$ as a function of $c_{\mathrm{MS}}$ and $\Omega$. We vary the $c_{\text {MS }}$ from 800 to $200 \mathrm{~m} \cdot \mathrm{s}^{-1}$, while keeping other parameters of Case I constant. It needs to be noted that the longitudinal speed of the coat $\left(185 \mathrm{~m} \cdot \mathrm{s}^{-1}\right)$ is much slower than that of the matrix $\left(1510 \mathrm{~m} \cdot \mathrm{s}^{-1}\right)$, which creates a precondition for the reduction of the effective speed.

From Fig. 4, it can be observed that the average value of the $c_{\mathrm{EL}}$ gradually reduces with the reduction of $c_{\mathrm{MS}}$. This is because the matrix becomes more 'rubbery' (higher Poisson's ratio) as $c_{\mathrm{MS}}$ decreases, making the reduction effect of the LRs more prominent. When the shear speed of the matrix is changed to $200 \mathrm{~m} \cdot \mathrm{s}^{-1}$, the effective speed of longitudinal waves in the composite maintains less than $700 \mathrm{~m} \cdot \mathrm{s}^{-1}$ regardless of frequency, except for the region where the resonance arises. Simultaneously, the Bragg scattering effects of the longitudinal waves gradually move to the lower frequency region with the reduction of $c_{\mathrm{MS}}$. This region can be identified by the contour lines which are almost parallel to the $y$-axis of the figure and equally spaced in frequency, since the $c_{\mathrm{EL}}$ of the composite increases linearly with frequency whenever Bragg scattering effects arise. As shown in Fig. 4, the Bragg scattering region for longitudinal waves moves from the frequency region $[0.87,1.05]$ when $c_{\mathrm{MS}}=800 \mathrm{~m} \cdot \mathrm{s}^{-1}$ to the region of $[0.55,0.78]$ when $c_{\mathrm{MS}}=200 \mathrm{~m} \cdot \mathrm{s}^{-1}$.

\section{Interaction between resonance and Bragg scattering in LRPCs}

For an LRPC with elastic matrix, the Bragg scattering band gap can only exist when both wave modes have strong scattering interference. Such influence will be more prominent for the following gap-coupled case. Therefore, we propose a new 3D LRPCs whose matrix alternates between elastic and fluid media periodically. Figure 5 shows a section view of such LRPC with alternating matrices.

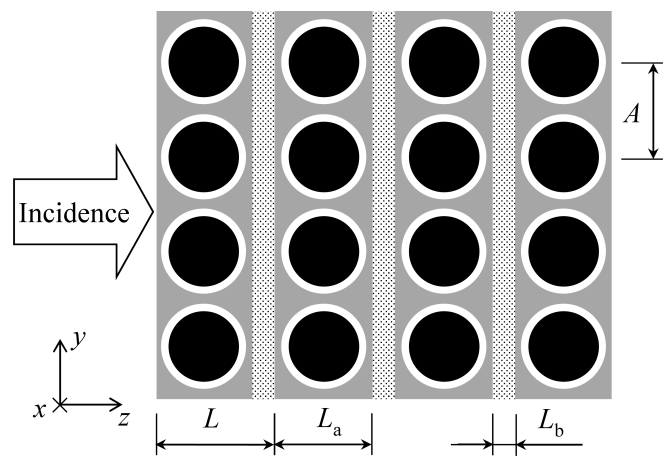

Fig. 5. The section view of the LRPC with alternating elastic and fluid matrices. The black circles represent resonators' cores; the white annuluses around the cores represent resonators' coats; the grey backgrounds represent the elastic matrix, the dotted areas represent the fluid matrix.

In this composite, LRs are periodically embedded in elastic medium, while each slab of elastic matrix is separated by fluid layers on both sides. The thickness of elastic slabs is referred to as $L_{\mathrm{a}}$, while the thickness of fluid layers as $L_{\mathrm{b}}$. The periodicity of such composite in $z$-direction $(L)$ is the sum of an elastic slab and a fluid layer. In order to obtain a Bragg scattering band gap 
at lower frequencies, the same basic LRPC is considered (refer to Case I), but with the shear speed of the elastic matrix $\left(c_{\mathrm{MS}}\right)$ reduced to $240 \mathrm{~m} \cdot \mathrm{s}^{-1}$. Besides, the lattice constant $(A)$ is taken to be $10 \mathrm{~mm}$, so as to reduce the impact of oblique propagating wave modes on the band structure. The fluid matrix is set as water with the layer thickness $\left(L_{\mathrm{b}}\right)$ to be $2 \mathrm{~mm}$, while the layer thickness of elastic matrix $\left(L_{\mathrm{a}}\right)$ is $30 \mathrm{~mm}$. The acoustic properties of water are $\rho_{\mathrm{W}}=1000 \mathrm{~kg} \cdot \mathrm{m}^{-3}$ and $c_{\mathrm{W}}=1480 \mathrm{~m} \cdot \mathrm{s}^{-1}$. This case is named as Case II.

The complex band structure of Case II is shown in Fig. 6a,b, where (a) shows the real part of the reduced $z$-component of complex wave-vectors, while (b) shows its imaginary part which will be nonzero when band gaps occur. Moreover, we also show the complex band structure of a counterpart case against the Case II in Fig. 6c,d, for the purpose of demonstrating the function of fluid layers. Such counterpart case has the same parameters as Case II except for substituting the water matrix with the elastic medium.

There are two band gaps in Fig. 6a,b for the considered frequency range. One is ranged from 0.22 to 0.31 , while the other from 0.40 to 0.90 . According to Ao and Chan (2009), it can be confirmed that the first band gap is induced by the local resonance. Because the real part of complex wave-vector $\left(\operatorname{Re}\left[k_{z} L\right]\right)$ jumps from $\pi$ to 0 at $\Omega=0.27$ inside the gap, and its imaginary trajectory has a cusp at the same frequency. On the contrary, the second band gap in the frequency range $[0.40,0.90]$ is provided by the Bragg scattering effect of the propagating waves inside the composite. The $\operatorname{Re}\left[k_{z} L\right]$ remains to be $\pi$ in the second band gap (dot- ted line in Fig. 6a), which implies that the wavelength of the propagating wave in $z$-direction is twice the layer thickness of the composite. Thus, constructive interference emerges among reflected waves from different layers as they are in-phase, and the propagating waves in the composite become evanescent waves. Meanwhile, the $\operatorname{Im}\left[k_{z} L\right]$ varies continuously and smoothly inside the band gap, and reaches its maximum near the midgap frequency.

By comparing the complex band structure of Case II with its counterpart, we can find that both cases have similar longitudinal dispersion curves in the same frequency region. But the LRPC with alternating matrices does not have shear dispersion curves, while the LRPC with elastic matrix has. This is because the water layers trap the scattered shear waves inside each elastic slab, while propagate the longitudinal waves from one side to the other. In contrast, the presence of shear dispersion curves in the counterpart case makes the band structure of the composite more complicated. The longitudinal forbidden band induced by Bragg scattering effect (range from 0.39 to 0.92 in Fig. 6c) is chopped off by the shear dispersion curves and separated into several band gaps.

In addition, when $\Omega>0.9$, the dispersion curves in both cases become more condensed than those in lower frequency region. Several flat dispersion curves can be observed in this area from Fig. 6a,c, which are induced by the oblique propagating waves. This is because the wavelength of sound is comparable with the lattice constant $(A)$ for such frequency region. Therefore, the oblique scattered waves in the composite can a)

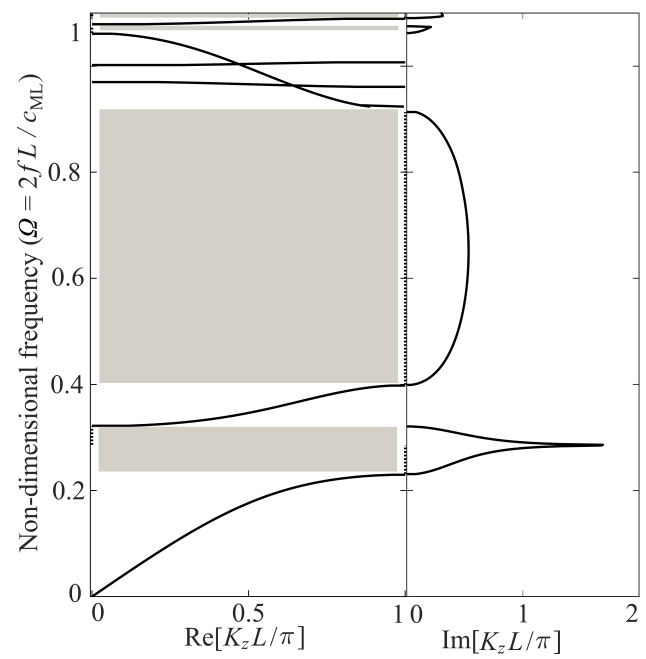

c)

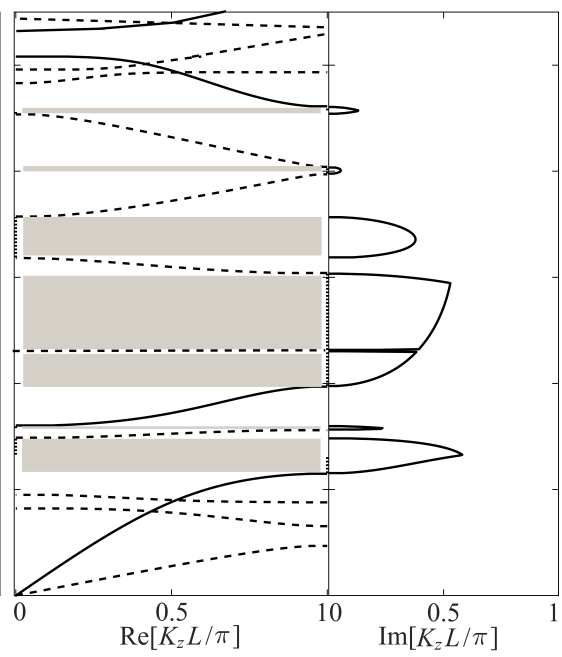

Fig. 6. a) The real part and b) the imaginary part of the complex band structure along z-direction for Case II; c) the real part and d) the imaginary part of the complex band structure for the counterpart of Case II with elastic matrix alone. The $y$-axis presents non-dimensional frequency, while the $x$-axes indicate the real and imaginary parts of the $z$-component of the reduced complex wave-vector. In (a) and (c), the solid curves represent longitudinal dispersion curves; the dashed curves represent shear dispersion curves; the shaded areas represent band gaps; while the dotted lines inside band gaps with the $\operatorname{Re}\left[k_{z} L\right]$ always stick to 0 or $\pi$ denote the real trajectory of the complex wave-vector. The solid curves in (b) and

(d) show the imaginary trajectory of the complex wave-vector, which will be nonzero only in band gaps. 
interfere constructively, which allows them to propagate through the composite efficiently.

The LRPC with alternating matrices already exhibits broad locally resonant and Bragg scattering gaps. To get further enhanced gaps by coupling resonance and Bragg scattering effects, we tuned the frequency of resonance by varying the shear speed of LR's coat based on Case II. This parameter can increase the resonant frequency of LRs without affecting the start frequency of Bragg scattering effect. Figure 7 shows the variation of imaginary part of complex wave-vectors as a function of the shear speed of coat and the nondimensional frequency.

In Fig. 7, there is a black curve inside the band gaps (painted areas). Such curve indicates the position of LR's resonant frequency as it is the peak attenuation of the resonance gap. It is readily to find out that with the increasing of coat's shear speed, the resonant frequency of LRs keeps moving to higher frequency, and the resonance gap moves closer to the Bragg scattering gap. Simultaneously, both the resonance and Bragg gaps are enhanced when they are closer to each other. When the resonant frequency overlaps the start frequency of the Bragg scattering gap, such composite yields a wide coupled band gap. For this case, such situation happens when coat's shear speed equals to $118.3 \mathrm{~m} \cdot \mathrm{s}^{-1}$.

Figure 8a,b show the complex band structure of the gap-coupled case, which is named as Case III. If the shear speed of coat keeps increasing, the resonance and

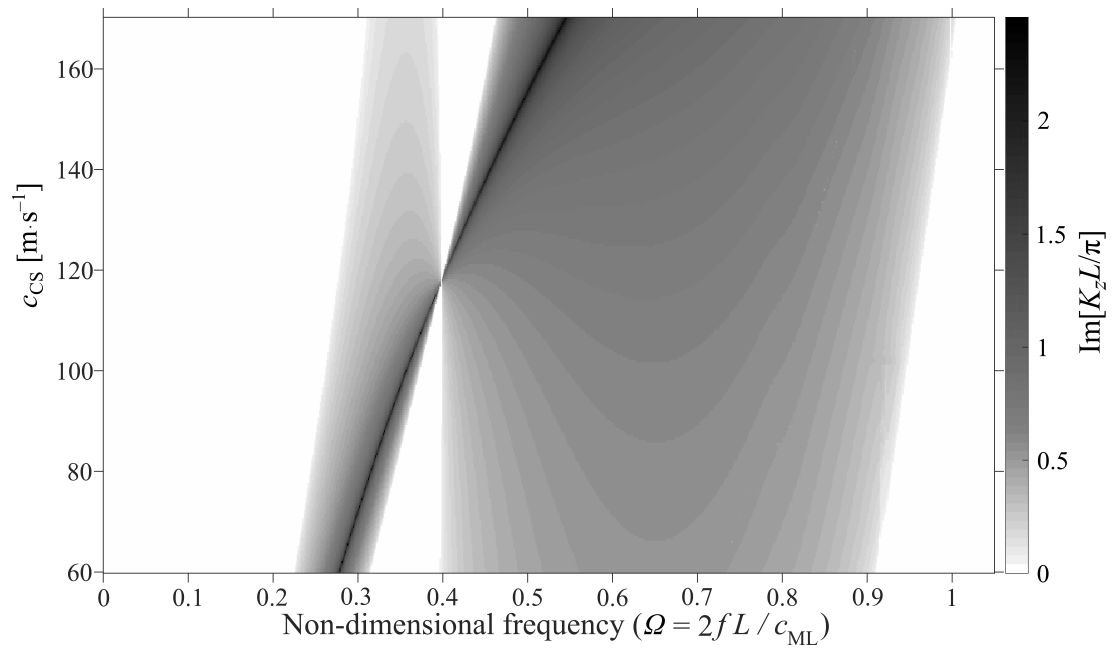

Fig. 7. The variation of imaginary part of complex wave-vectors accompanied by increasing the shear speed of coat based on Case II. The x-axis represents the non-dimensional frequency $(\Omega)$; the $y$-axis represents the shear speed of LR's coat $\left(c_{\mathrm{CS}}\right)$; the grey scale indicates the imaginary part of the complex wave-vector. White areas in the graph denotes the pass bands as $\operatorname{Im}\left[k_{z} L / \pi\right]=0$, while painted areas means the band gaps with black implying maximum attenuation.

a)

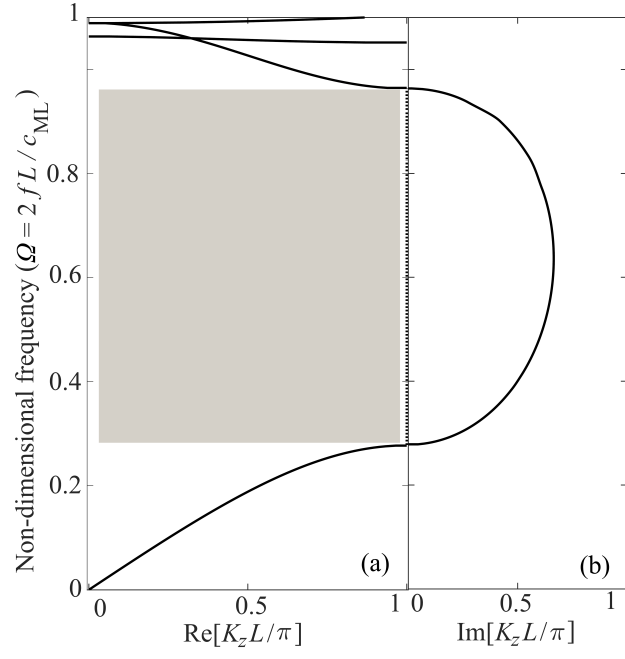

c)

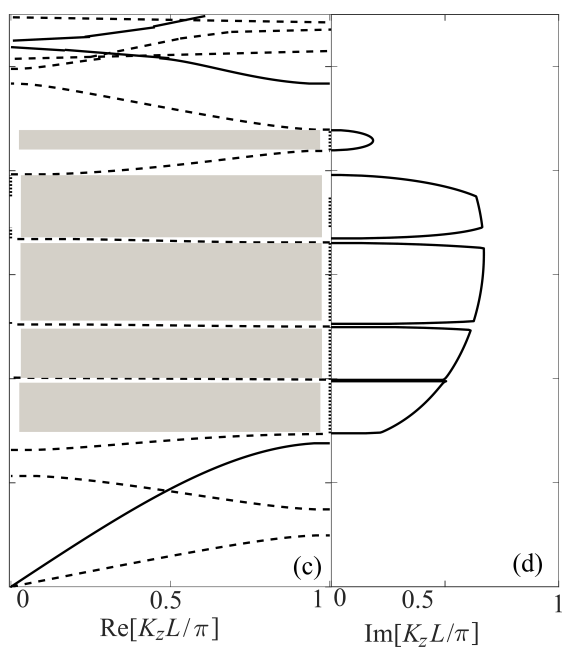

Fig. 8. a) The real part and b) the imaginary part of the complex band structure for the gap-coupled LRPC (shear speed of coat is $117.7 \mathrm{~m} \cdot \mathrm{s}^{-1}$ ) with alternative solid and water matrices; c) the real part and (d) the imaginary part of the complex band structure for the gap-coupled LRPC with elastic matrix alone (shear speed of coat is $116.1 \mathrm{~m} \cdot \mathrm{s}^{-1}$ ). The meaning of axes and curves are the same as those in Fig. 6. 
Bragg gaps will decouple and switch their positions. At this time, the end frequency of the Bragg gap (located at $\Omega=0.4$ ) will hold its position where the start frequency was before the coupling, while the resonance gap keeps rising to higher frequencies. All the phenomena described here are similar to the gap coupling behaviour of the 1D LRES (XIAO et al., 2011), except for some unique features existing in 3D LRPC, such as oblique propagating waves in the composite and the shear dispersion curves for LRPC with an elastic matrix. To demonstrate the advantage of the LRPC with alternating matrices, Fig. 8c,d illustrates the band structure of a counterpart LRPC for the gapcoupled case which substituted the water matrix with elastic medium. Due to the changing of matrix, the condition for the gap-coupled case is slightly different with Case III. For the counterpart with elastic matrix alone, the coupled gap emerges when the shear speed of coat is $116.1 \mathrm{~m} \cdot \mathrm{s}^{-1}$.

Comparing subplots (a) and (c) in Fig. 8, we can discover that the water layers inserted between solid slabs not only make the dispersion relation of composite much simpler, but also maximize the frequency range of the coupled gap by eliminating the shear propagating waves in the composite. As shown in Fig. 8a,b, the composite demonstrates a huge coupled gap extending from $\Omega=0.27$ to 0.97 , with its attenuation $\left(\operatorname{Im}\left[k_{z} L\right]\right)$ varying smoothly through this region. In contrast, although two kinds of gaps are coupled together for the LRPC with elastic matrix when the shear speed of coat is $116.1 \mathrm{~m} \cdot \mathrm{s}^{-1}$, the shear dispersion curves split the coupled gap into several pieces, which is shown in Fig. 8c,d. Someone may suppose an LRPC with local resonators periodically embedded in fluid matrix will have a better performance for the coupling of band gaps, since shear waves can never be stimulated in the matrix. However, the result is not as good as the expectation. A major defect of such proposal is that the resonant modes of LRs are decoupled with the scattered waves in the fluid matrix. As a result, the enhancement of band gaps is limited when trying to couple them.

Overall, the LRPC with alternating matrices proposed in this paper inherits the merits of both elastic and fluid matrix cases. It exhibits a broad subwavelength coupled gap with simple dispersion relations. Such proposal also excels at the application of underwater acoustic materials. Since the elastic slabs are separate with each other, we can conveniently adjust the frequency region of the coupled gap by substituting other elastic slabs with different LRs, as well as varying the distance between slabs. Furthermore, multiple coupled gaps are designable by using different resonators in different elastic slabs. If the thickness of the composite is not crucial, one can easily increase the distance between elastic slabs so as to achieve the sound blocking performance in the super sub-wavelength region without using bulky elastic matrices.

\section{Conclusion}

The aim of this paper is to optimise the subwavelength band gaps of three-dimensional (3D) locally resonant phononic crystals (LRPCs), which ideally apply for the underwater acoustic materials. Using the complex reflection and transmission coefficients of such composite to retrieve the effective acoustic speeds $\left(c_{\mathrm{E}}\right)$, we analysed the influence of local resonators (LRs) on the acoustic properties of LRPCs. It has been observed that the $c_{\mathrm{E}}$ will drop to zero when local resonance arise, and will increase monotonically when Bragg scattering effects occur in the composite. Furthermore, analysis reveals that LRs are capable of reducing the effective acoustic speeds of the composite significantly when the matrix is a low-shear-speed elastic material. This provides a prospective method to reduce the frequency region of Bragg scattering effects and hence, to couple resonance and Bragg gaps in sub-wavelength zone.

Based on this analysis, we proposed a specific LRPC with alternating elastic and fluid matrices, whose Bragg scattering effects are already close to the local resonance in frequency. The fluid matrix traps the scattered shear waves in each elastic matrix and thus, prohibits the shear waves from propagating in the composite. Such proposal is also suit for the application of underwater acoustic materials. Since the elastic slabs are separate from each other, one can conveniently adjust the arrangement of the structure for tuning the gaps' frequencies. By tuning the frequency of resonance, we can create a huge coupled gap in the sub-wavelength region for the proposed case. Such work offers a new technique for broadening the lowfrequency band gaps of 3D LRPCs, which is potential for designing underwater acoustic materials.

\section{Acknowledgments}

This research has been funded by the National Natural Science Foundation of China under Grant Nos. 11504427 and 11404405, and by the Youth Foundation of Logistical Engineering University under Grant No. YQ14-421001.

\section{References}

1. Ao X., Chan C. (2009), Complex band structures and effective medium descriptions of periodic acoustic composite systems, Physical Review B, 80, 235118.

2. Elford D., Chalmers L., Kusmartsev F., SwalLOWE G. (2011), Matryoshka locally resonant sonic 
crystal, Journal of the Acoustical Society of America, 130, 2746.

3. FANO U. (1961), Effects of Configuration Interaction on Intensities and Phase Shifts, Physical Review, 124, 1866.

4. Fokin V., Ambati M., Sun C., Zhang X. (2007), Method for retrieving effective properties of locally resonant acoustic metamaterials, Physical Review B, 76, 144302 .

5. Goffaux C., SÁnchez-Dehesa J. (2003), Two-dimensional phononic crystals studied using a variational method: Application to lattices of locally resonant materials, Physical Review B, 67, 144301.

6. Goffaux C., SÁnchez-Dehesa J., Yeyati A., LamBin P., Khelif A., Vasseur J., Djafari-Rouhani B. (2002), Evidence of Fano-like interference phenomena in locally resonant materials, Physical Review Letter, 88, 225502.

7. HiRSEKORN M. (2004), Small-size sonic crystals with strong attenuation bands in the audible frequency range, Applied Physics Letters, 84, 3364-3366.

8. Kuang W., Hou Z., Liu Y. (2004), The effects of shapes and symmetries of scatterers on the phononic band gap in 2D phononic crystals, Physics Letters A, 332, 481-490.

9. Kushwaha M., Halevi P., Dobrzynski L., DjafaRI-Rouhani B. (1993), Acoustic band structure of periodic elastic composites, Physical Review Letter, 71, 2022 .

10. Larabi H., Pennec Y., Djafari-Rouhani B., VasSEUR J. (2007), Multicoaxial cylindrical inclusions in locally resonant phononic crystals, Physical Review E, 75, 066601.

11. Liu Z., Chan C., Sheng P. (2002), Three-component elastic wave band-gap material, Physical Review B, 65, 165116.
12. Liu Z., Chan C., Sheng P. (2005), Analytic model of phononic crystals with local resonances, Physical Review $\mathrm{B}, \mathbf{7 1}, 014103$.

13. LiU Z., Zhang X., MaO Y., Zhu Y., Yang Z., Chan C., Sheng P. (2000), Locally resonant sonic materials, Science, 289, 1734.

14. Sainidou R., Stefanou N., Psarobas I.E., ModiNos A. (2005), A layer-multiple-scattering method for phononic crystals and heterostructures of such, Computer Physics Communications, 166, 197-240.

15. Sigalas M., Economou E. (1992), Elastic and acoustic wave band structure, Journal of Sound and Vibration, 158, 377 .

16. Wang G., Shao L., Liu Y., Wen J. (2006), Accurate evaluation of lowest band gaps in ternary locally resonant phononic crystals, Chinese Physics, 15, 18431848.

17. Wen J., Zhao H., Lv L., Yuan B., Wang G., WEN X. (2011), Effects of locally resonant modes on underwater sound absorption in viscoelastic materials, Journal of the Acoustical Society of America, 130, 1201-1208.

18. Xiao Y., Mace B., Wen J., Wen X. (2011), Formation and coupling of band gaps in a locally resonant elastic system comprising a string with attached resonators, Physics Letters A, 375, 1485.

19. Zhao H., Liu Y., Wang G., Wen J., Yu D., Han X., WEN X. (2005), Resonance modes and gap formation in a two-dimensional solid phononic crystal, Physical Review B, 72, 012301.

20. Zhao H., Liu Y., Wen J., Yu D., Wen X. (2007), Tri-component phononic crystals for underwater anechoic coatings, Physics Letters A, 367, 224-232.

21. ZHOU X., HU G. (2009), Analytic model of elastic metamaterials with local resonances, Physical Review B, 79, 195109. 